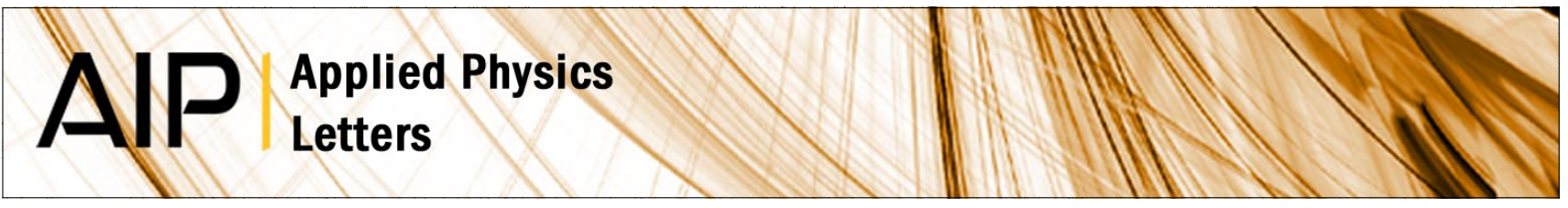

\title{
Saturation effects in femtosecond laser ablation of silicon-on-insulator
}

Hao Zhang, D. van Oosten, D. M. Krol, and J. I. Dijkhuis

Citation: Appl. Phys. Lett. 99, 231108 (2011); doi: 10.1063/1.3666423

View online: http://dx.doi.org/10.1063/1.3666423

View Table of Contents: http://apl.aip.org/resource/1/APPLAB/v99/i23

Published by the American Institute of Physics.

\section{Related Articles}

Production and acceleration of ion beams by laser ablation

Rev. Sci. Instrum. 83, 02B717 (2012)

Proton emission from a laser ion source

Rev. Sci. Instrum. 83, 02B310 (2012)

Optical diagnosis and theoretical simulation of laser induced lead plasma spectrum

Phys. Plasmas 19, 013302 (2012)

Effect of air breakdown with a focusing lens on ultrashort laser ablation

Appl. Phys. Lett. 99, 234104 (2011)

Gas dynamic effects on formation of carbon dimers in laser-produced plasmas

Appl. Phys. Lett. 99, 131506 (2011)

\section{Additional information on Appl. Phys. Lett.}

Journal Homepage: http://apl.aip.org/

Journal Information: http://apl.aip.org/about/about_the_journal

Top downloads: http://apl.aip.org/features/most_downloaded

Information for Authors: http://apl.aip.org/authors

\section{ADVERTISEMENT}

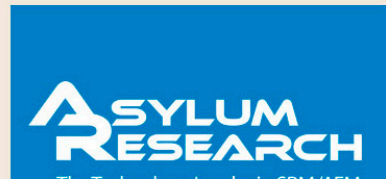

The Technology Leader in SPM/AFM

Register Now at

www.asylumresearch.com

\section{Free AFM Webinar February 22 Register Now}

"Smaller and Quieter: Ultra-High Resolution AFM Imaging"

With Jason Cleveland, AFM pioneer, inventor and Asylum Research co-founder

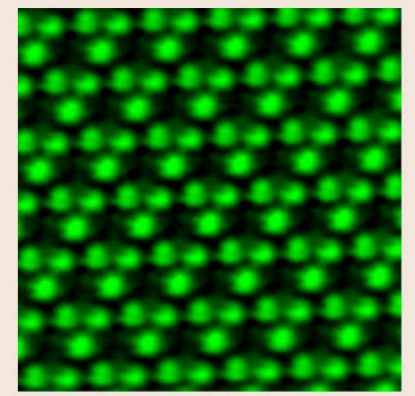




\title{
Saturation effects in femtosecond laser ablation of silicon-on-insulator
}

\author{
Hao Zhang, ${ }^{\text {a) }}$ D. van Oosten, D. M. Krol, ${ }^{\text {b) }}$ and J. I. Dijkhuis \\ Debye Institute for Nanomaterials Science, Utrecht University, P.O. Box 80000, 3508 TA Utrecht, \\ The Netherlands
}

(Received 3 October 2011; accepted 15 November 2011; published online 8 December 2011)

\begin{abstract}
We report a surface morphology study on single-shot submicron features fabricated on silicon on insulator by tightly focused femtosecond laser pulses. In the regime just below single-shot ablation threshold nano-tips are formed, whereas in the regime just above single-shot ablation threshold, a saturation in the ablation depth is found. We attribute this saturation by secondary laser absorption in the laser-induced plasma. In this regime, we find excellent agreement between the measured depths and a simple numerical model. When the laser fluence is further increased, a sharp increase in ablation depth is observed accompanied by a roughening of the ablated hole. (C) 2011 American Institute of Physics. [doi:10.1063/1.3666423]
\end{abstract}

Femtosecond laser ablation of solid materials has become an increasingly common tool for precise machining of micron and submicron features in both dielectrics ${ }^{1}$ and semiconductors. $^{2,3}$ Silicon, as the most important element in today's semiconductor industry, has drawn a lot of attention from researchers to study both the fundamental response to ultrashort pulsed laser radiation ${ }^{4-7}$ and its potential application in direct laser writing of photonic devices, ${ }^{2}$ laser fabrication of nano-tips on thin silicon films, ${ }^{8,9}$ direct laser amorphization of silicon, ${ }^{10,11}$ and microspiked or black silicon. ${ }^{12,13}$

Photonic crystal waveguide slabs are usually made out of silicon-on-insulator (SOI) by electron-beam and x-ray lithography, as well as focused ion beam (FIB) milling. ${ }^{2}$ The emergence of femtosecond laser microfabrication makes sub diffraction-limited features directly fabricated on SOI wafer possible. For instance, Li et al. reported the fabrication of a photonic bandpass filter at $1550 \mathrm{~nm}$ by femtosecond direct laser ablation. ${ }^{2}$ Unlike the case for bulk silicon, few studies on ultrafast laser machining of SOI wafers have been reported. ${ }^{2,10}$ Furthermore, most studies in silicon ${ }^{3,7}$ are done in a relatively high fluence regime. Therefore, these studies shed limited light on the ablation behavior around the ablation threshold.

In this letter, we present a study on submicron features fabricated on the front surface of SOI $(200 \mathrm{~nm}$ c-Si layer on $1 \mu \mathrm{m}$ thick $\mathrm{SiO}_{2}$ layer) by tightly focused femtosecond laser pulses. We determine the surface morphology and ablation depth using atomic force microscopy (AFM) in a fluence range around the ablation threshold and identify three modification regimes. Subsequently, we model the ablation behavior around the ablation threshold, taking into account one-(OPA) and two-photon absorption (TPA) and secondary absorption by the optically generated carriers. Crucially, we also take full account of the effect of transient reflectivity during the laser pulse itself.

An amplified Ti:Sapphire femtosecond laser (Hurricane, Spectra Physics Inc.) operating in single-shot mode was used to produce pulses centered around $800 \mathrm{~nm}$, with an initial

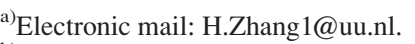

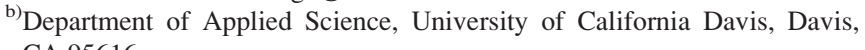
CA 95616.
}

pulse duration of 100 fs. These linearly polarized pulses were focused on the $\mathrm{c}-\mathrm{Si}$ layer by a NA $=0.8$ objective lens (Nikon CFI60, 100×). We estimate that group velocity dispersion and propagation time difference across the aperture will increase the pulse duration to up to $150 \mathrm{fs}{ }^{14}$ Three-axis high-precision motorized translation stages (T-LSM025A, Zaber Technologies Inc.) were used to find the exact focal position of the objective and scan the sample to provide a fresh surface for every shot. A charge coupled device (CCD) camera (Qimaging RETIGA 1300) was utilized to allow in-situ monitoring of the sample surface. Special care was taken to keep the sample surface in focus during the whole experiment, since the depth of field of the CFI60 objective is smaller than $1 \mu \mathrm{m}$. The focused beam diameter is measured to be $1.2 \mu \mathrm{m}$ (at $1 / e^{2}$ maximum intensity) by analyzing the CCD image of the focused spot. The pulse energy was varied in the range $0.09 \mathrm{~J} / \mathrm{cm}^{2}<F<0.7 \mathrm{~J} / \mathrm{cm}^{2}$ using a rotating $\lambda / 2$ plate followed by a polarizing beam splitter.

Single-shots at various laser fluences were launched on the top c-Si layer of the SOI. Experiments were repeated 3 times to test the reproducibility and to get an estimate of experimental error. All experiments were conducted in ambient air and at room temperature. An atomic force microscope (Digital Instruments) operating in tapping mode was used to measure the detailed surface morphology of the lasermodified sample. As shown in Fig. 1, three types of surface morphology, nano-tips (Fig. 1(a)), smooth craters (Fig. 1(b)), and rough craters (Fig. 1(c)) can be clearly identified. In Fig. 2, the depth of the craters, defined by the distance from the bottom of the crater to the flat sample surface, is plotted as a function of laser fluence. As can be seen, the crater depth first saturates at $50 \mathrm{~nm}$ and then quickly increases to about $150 \mathrm{~nm}$. We can identify three regimes of modification: (a) nano-tip formation regime $\left(0.13 \mathrm{~J} / \mathrm{cm}^{2}<F<0.22 \mathrm{~J} / \mathrm{cm}^{2}\right)$, (b) gentle ablation regime $\left(0.22 \mathrm{~J} / \mathrm{cm}^{2}<F<0.35 \mathrm{~J} / \mathrm{cm}^{2}\right)$, and (c) heavy ablation regime $\left(F>0.35 \mathrm{~J} / \mathrm{cm}^{2}\right)$. These can be related to the various types of surface morphology or to the crater depths. Single-shot surface morphology modification is observed at laser fluences above $0.13 \mathrm{~J} / \mathrm{cm}^{2}$. In the fluence range from $0.13 \mathrm{~J} / \mathrm{cm}^{2}$ to $0.18 \mathrm{~J} / \mathrm{cm}^{2}$, nano-tips are formed, with a width of $150 \mathrm{~nm}$ and $20 \mathrm{~nm}$ in height (Fig. 1(d)). The same experiment was performed in bulk silicon but here this 

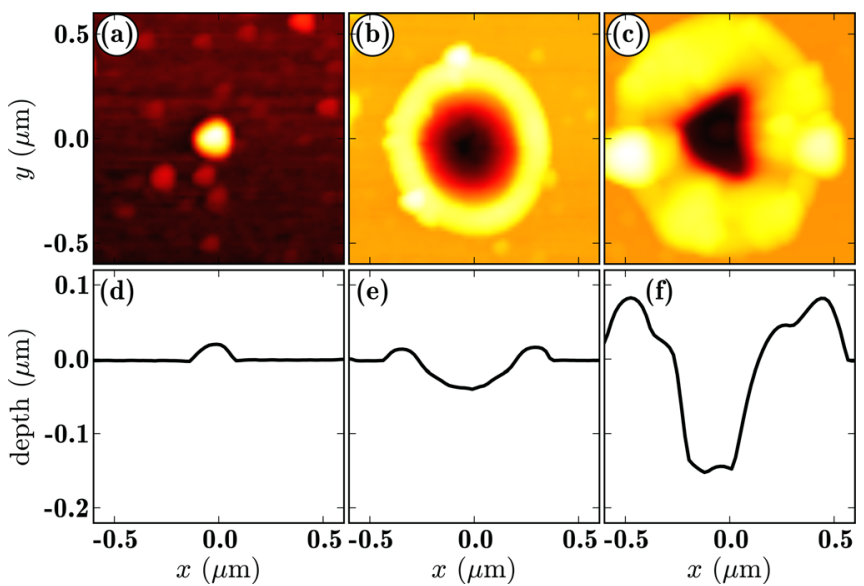

FIG. 1. (Color online) Typical AFM images (top row) and cross-sections (bottom row) of single-shot submicron features on SOI wafer for (a) and (d) nano-tip formation (fluence $F \sim 0.13 \mathrm{~J} / \mathrm{cm}^{2}$ ), (b) and (e) gentle ablation $\left(F \sim 0.3 \mathrm{~J} / \mathrm{cm}^{2}\right)$, and (c) and (f) heavy ablation regime $\left(F \sim 0.6 \mathrm{~J} / \mathrm{cm}^{2}\right)$. Note that the three AFM images have a different color scaling.

nano-tip formation regime is missing, indicating that the particular layered arrangement of the SOI wafer is crucial for the formation of nano-tips. In fact, in Ref. 8, Georgiev et al. reported similar nano-tip formation in SOI fabricated by a nano-second laser. ${ }^{8,9}$ These authors attribute the formation of nano-tips to the predominantly lateral dissipation of heat from the laser-heated spot. The nano-tip formation regime in our experiment suggests that thermal effects may not be completely ignored even for a femtosecond pulse. Turning now to the ablation regimes, the data in Fig. 2 show that in the gentle ablation regime, the ablation depth saturates at about $50 \mathrm{~nm}$, before it steeply increases in the heavy ablation regime. Inspection of the AFM images shows that, in the gentle ablation regime, smooth craters are formed (Fig. 1(b)), whereas in the heavy ablation regime, the craters become rough and deformed (Fig. 1(c)). As we will show, a detailed knowledge of laser absorption in excited silicon is crucial to understand this saturation effect. The creation of electron-hole pairs via absorption of ultra short pulse radiation is mainly due to OPA and TPA. ${ }^{3,4,7}$ We show that the secondary laser absorption

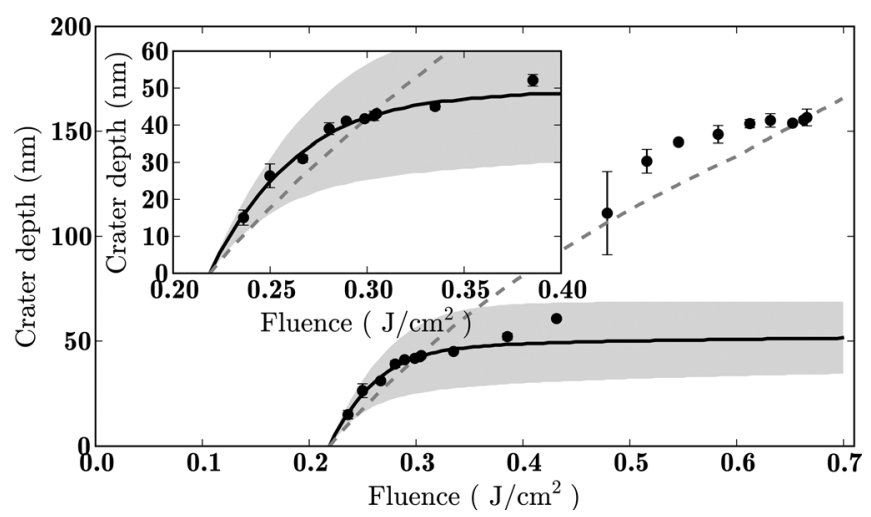

FIG. 2. The data points indicate the crater depth measured on SOI, averaged over three experiments. The error bars indicate the standard deviation in measured crater depth. Solid curve shows the calculated crater depth using a $136 \mathrm{fs}$ pulse duration. The shaded area indicates the effect of the pulse duration on the ablation depth. Dashed curve shows the crater depth calculated only taking OPA and TPA into account. The inset shows a magnified view of the gentle ablation regime. due to the interaction between the incident pulses and the dense electron-hole plasma excited via OPA and TPA must be taken into account to explain the saturation of the ablation depth observed in the experimental data. Inside the material, laser intensity is described by a Lambert-Beer like law, ${ }^{7}$

$$
\frac{\partial I}{\partial x}=-\left(\alpha_{0}+\alpha_{\text {Drude }}\right) I-\beta I^{2},
$$

where $I=(1-R) I_{0}, R$ the transient surface reflectivity, and $I_{0}$ the incident intensity. The OPA coefficient $\alpha_{0}=1021 \mathrm{~cm}^{-1}$ (at $\lambda=800 \mathrm{~nm}$ ) is taken from Ref. 7, and the TPA coefficient $\beta=1.85 \mathrm{GW} / \mathrm{cm}$ is taken from Bristow et al. ${ }^{15}$ The secondary laser absorption in the excited electron-hole plasma is described by a time and space dependent extra absorption coefficient $\alpha_{\text {Drude. }}$. By considering the creation of electron-hole pairs via OPA and TPA and the diffusion of the created carriers through ambipolar diffusion, the balance equation for carrier number density $\mathrm{N}$ can be written as follows: ${ }^{4,7}$

$$
\frac{\partial N}{\partial t}+\nabla \cdot\left(-D_{0} \nabla N\right)=\frac{\alpha_{0} I}{\hbar \omega}+\frac{\beta I^{2}}{2 \hbar \omega},
$$

where $D_{0}$ is the coefficient of ambipolar diffusivity $\left(18 \mathrm{~cm}^{2} / \mathrm{s}\right)$ (Ref. 7) and $\hbar \omega$ is the photon energy. Recombination and impact ionization can be safely ignored since the time period of our computation is only $600 \mathrm{fs}$. The reflectivity $R$ as well as the absorption $\alpha_{\text {Drude }}$ in the excited plasma change during the pulse due to the time and space dependence of the carrier number density $N .^{7}$ The complex refractive index $n$ of the highly excited silicon is calculated by a simple Drude model of the dense electron-hole plasma according to the following expression: ${ }^{4,7}$

$$
n=\sqrt{\varepsilon_{\mathrm{c}-\mathrm{Si}}-\frac{\omega_{p}^{2}}{\omega^{2}+i \omega / \tau_{d}}},
$$

where $\varepsilon_{\mathrm{c}-\mathrm{Si}}$ is the dielectric constant of crystalline silicon, $\omega$ is the angular frequency of the incident pulse, and the damping time $\tau_{\mathrm{d}}$ is chosen at $1.1 \mathrm{fs}$ considering high carrier-carrier collisions. ${ }^{4}$ The plasma frequency $\omega_{p}$ is calculated from the transient carrier density as $\omega_{p}=\left(4 \pi N e^{2} / m^{*}\right)^{1 / 2}$, where $m^{*}$ is the electron effective mass taken as $m^{*}=0.18 m_{e}{ }^{4}$ The laser absorption in the plasma $\alpha_{\text {Drude }}$ is given by $\alpha_{\text {Drude }}=4 \pi \kappa / \lambda$, where $\kappa$ is the imaginary part of $n$. The transient reflectivity of the plasma layer excited by the incident pulse can be calculated using the transfer matrix method. ${ }^{16}$ By extrapolating the measured crater depth to zero (Fig. 2), a single shot ablation threshold $F_{\text {av }}=0.22 \mathrm{~J} / \mathrm{cm}^{2}$ is deduced which is close to the threshold value measured by other groups $\left(0.2 \mathrm{~J} / \mathrm{cm}^{2}\right){ }^{17,18}$ We propose following a simple but crucial model to determine the ablation depth. The coupled equations (1)-(3) are solved numerically by a fully implicit finite difference scheme, and the crater depth is modeled by calculating the region where the laser fluence is larger than the threshold value of $F_{\text {peak }}=0.321 \mathrm{~J} / \mathrm{cm}^{2}$. This peak fluence is the fluence inside the material at the center of the laser spot $\left(2 F_{\text {av }}\right)$ corrected for the part of the pulse that is transiently reflected by the excited silicon. 
The data points in Fig. 2 show the measured crater depth as a function of fluence. The pulse duration was used as a fit parameter. The best correspondence was found with a pulse duration of $136 \mathrm{fs}$, which is consistent with our estimates. The solid line is the result of the model calculation using that pulse duration. To indicate the effect of the uncertainty in the in-situ pulse duration, the shaded area indicates the result when pulse durations between $100 \mathrm{fs}$ and $172 \mathrm{fs}$ are used. As is seen in Fig. 2, agreement between calculation and measurement is excellent for laser fluences below $0.35 \mathrm{~J} / \mathrm{cm}^{2}$. We have found the same saturation effect in bulk silicon, indicating the saturation is not caused by the particular layered structure of the SOI wafer. In Ref. 3, AFM measurements on ablation depth using pulse fluence $F>0.7 \mathrm{~J} / \mathrm{cm}^{2}$ are carried out. The authors fit the ablation depth with a simple logarithmic function, as is appropriate when only taking OPA and TPA into account. As is seen in Fig. 2, such a logarithmic fit (dashed grey curve) leads to a rather poor correspondence close to the threshold. Furthermore, this logarithmic fit can only be obtained using an unrealistically large TPA coefficient of $\beta=29 \mathrm{~cm} / \mathrm{GW}$. Based on this consideration, we contribute the saturation in ablation depth to the secondary laser absorption in the laser-induced plasma. For laser fluences beyond the gentle ablation regime, our model no longer describes the data, which suggests that the ablation depth in the heavy ablation regime is no longer determined by the effective optical penetration depth, but rather by the effective energy penetration depth. ${ }^{3}$ However, identifying the exact mechanism for the departure from the model requires further study.

In conclusion, submicron features were directly fabricated on SOI wafer by tightly focused single-shot femtosecond laser pulses. In the regime below single-shot ablation threshold, nano-tips are formed. In the regime just above single-shot ablation threshold, we found a saturation in ablation depth which can be understood by secondary laser absorption in the laser-induced plasma. We expect that this saturation can be used as an effective depth-stop in laser processing. Such a depth-stop is technically very beneficial for laser milling of structure, especially in stratified media such as SOI. The calculated ablation depth agrees excellently with the measured value in this regime. Beyond this regime, the ablation depth exhibits a sharp increase, which requires further study.

We thank C. R. de Kok and P. Jurrius for discussions and technical assistance. D.v.O. gratefully acknowledges the support by the NWO (VIDI grant). H.Z. acknowledges the financial support from China Scholarship Council.

${ }^{1}$ E. G. Gamaly, S. Juodkazis, K. Nishimura, H. Misawa, B. Luther-Davies, L. Hallo, P. Nicolai, and V. T. Tikhonchuk, Phys. Rev. B 73, 214101 (2006).

${ }^{2}$ M. Li, K. Mori, M. Ishizuka, X. Liu, Y. Sugimoto, N. Ikeda, and K. Asakawa, Appl. Phys. Lett. 80, 216 (2003).

${ }^{3}$ D. J. Hwang, C. P. Grigoropoulos, and T. Y. Choi, J. Appl. Phys. 99, 083101 (2006).

${ }^{4}$ K. Sokolowski-Tinten and D. von der Linde, Phys. Rev. B 61, 2643 (2000).

${ }^{5}$ P. Stampfli and K. H. Bennemann, Phys. Rev. B 46, 10686 (1992).

${ }^{6}$ H. M. van Driel, Phys. Rev. B 35, 8166 (1987).

${ }^{7}$ T. Y. Choi and C. P. Grigoropoulos, J. Appl. Phys. 92, 4918 (2002); T. Y. Choi and C. P. Grigoropoulos, J. Heat Transfer 126, 723 (2004).

${ }^{8}$ D. G. Georgiev, R. J. Baird, I. Avrusky, G. Auner, and G. Newaz, Appl. Phys. Lett. 84, 4881 (2004).

${ }^{9}$ J. Eizenkop, I. Avrutsky, G. Auner, D. G. Georgiev, and V. Chaudhary, J. Appl. Phys. 101, 094301 (2007); J. Eizenkop, I. Avrutsky, D. G. Georgiev, and V. Chaudhary, J. Appl. Phys. 103, 094311 (2008).

${ }^{10}$ J. Jia, M. Li, and C. V. Thompson, Appl. Phys. Lett. 84, 3205 (2004).

${ }^{11}$ A. Kiani, K. Venkatakrishnan, and B. Tan, J. Appl. Phys. 108, 074907 (2010).

${ }^{12}$ M. Y. Shen, C. H. Crouch, J. E. Carey, R. Younkin, E. Mazur, M. Sheehy, and C. M. Friend, Appl. Phys. Lett. 82, 1715 (2003); M. Y. Shen, C. H. Crouch, J. E. Carey, R. Younkin, and E. Mazur, Appl. Phys. Lett. 85, 5694 (2004).

${ }^{13}$ T.-H. Her, R. J. Finlay, C. Wu, S. Deliwala, and E. Mazur, Appl. Phys. Lett. 73, 1673 (1998)

${ }^{14}$ P. E. Hänninen and S. W. Hell, Bioimaging 2, 117 (1994).

${ }^{15}$ A. D. Bristow, N. Rotenberg, and H. M. Van Driel, Appl. Phys. Lett. 90, 191104 (2007).

${ }^{16} \mathrm{M}$. Born and E. Wolf, Principles of Optics (Cambridge University Press, Cambridge, 1999).

${ }^{17}$ H. O. Jeschke, M. E. Garcia, M. Lenzner, J. Bonse, J. Kruger, and W. Kautek, Appl. Surf. Sci. 197-198, 839 (2002).

${ }^{18}$ P. P. Pronko, P. A. VanRompay, C. Horvath, F. Loesel, T. Juhasz, X. Liu, and G. Mourou, Phys. Rev. B 58, 2387 (1998). 